\title{
GPFIS-CONTROL: A GENETIC FUZZY SYSTEM FOR CONTROL TASKS
}

\author{
Adriano S. Koshiyama, Marley M. B. R. Vellasco and Ricardo Tanscheit \\ Department of Electrical Engineering, Pontifical Catholic University of Rio de Janeiro Rua Marqus de So Vicente, \\ 225, Gvea - Rio de Janeiro, RJ, Brazil
}

\begin{abstract}
This work presents a Genetic Fuzzy Controller (GFC), called Genetic Programming Fuzzy Inference System for Control tasks (GPFIS-Control). It is based on Multi-Gene Genetic Programming, a variant of canonical Genetic Programming. The main characteristics and concepts of this approach are described, as well as its distinctions from other GFCs. Two benchmarks application of GPFIS-Control are considered: the Cart-Centering Problem and the Inverted Pendulum. In both cases results demonstrate the superiority and potentialities of GPFIS-Control in relation to other GFCs found in the literature.
\end{abstract}

\section{Introduction}

Fuzzy Logic Controllers (FLCs) [1,2] have been extensively used as an alternative to manipulate and describe complex systems when traditional control methods do not provide viable solutions. FLCs have the capacity of modeling systems by using fuzzy "if-then" rules, normally provided by an expert. Classical fuzzy logic approaches employ either a Mamdani-type Fuzzy Inference System (FIS) [2-3] or a Takagi-Sugeno (TSK) FIS [4-5] and both have different parameters that must be tuned in order to obtain the best performance, such as rule base, membership function parameters, etc. These parameters can be tuned manually by an expert or automatically by employing a learning approach. In this respect, this work considers Genetic Fuzzy Systems [3,6], or, more specifically, Genetic Fuzzy Controllers.

In Genetic Fuzzy Controllers (GFC) the automatic learning and tuning of parameters is based on a Genetic-based Meta-Heuristic (GBMH). Some previous works have considered FLCs embedded with a Genetic Algorithm (GA) to tune membership function parameters [7-8] or to search for concise fuzzy rule bases [9-10]. More recently, some works have explored Genetic Programming (GP) to build an FLC by using methodologies and concepts similar to those employed on a GA based FLC [11-12].

In general, it is advantageous to use a GBMH exclusively to search for the FLC best configuration. In this perspective, the meta-heuristic is seen as a tool to build an FLC and not as a mechanism that may change reasoning. Still, in frameworks with a high level of hybridization, in which a genetic-based meta-heuristic has a higher participation, it may be possible to obtain better accuracy. Examples are Neuro-Fuzzy models [13,14], where Neural Networks play an important role in the hybrid architecture, enabling high accuracy and fast convergence.

This work proposes a new GFC called Genetic Programming Fuzzy Inference System for Control tasks (GPFIS-Control). It makes use of Multi-Gene Genetic Programming [15-16] for extracting knowledge from the plant. The resulting architecture should: (1) automatically tune the FLC parameters; (2) make the plant output reach the setpoint as fast as possible; (3) provide linguistic comprehension 
for each FLC action; and (4) be easy to implement.

This paper is organized as follows: Section 2 describes related works on GFC and considers some applications involving GP. Section 3 describes Multi-Gene Genetic Programming and its basic differences from standard Genetic Programming strategy. Section 4 presents the proposed GPFIS-Control in detail. Case studies are considered in Section 5 and section 5 concludes the work.

\section{Related Works}

The first attempt to build an FLC by using GBMH algorithms was presented in [7], where a GA was used to tune membership functions parameters of input and output variables. Subsequently, many other researchers have employed evolutionary algorithms, mostly GA, to tune FLC parameters and search for concise rule bases [17-19].

Several works can be found in the GFC area, such as [9], which presents an evolutionary procedure to modify rules, initially set by an expert, for a Mamdani type FLC. In [20], membership functions, rule sets and consequent types (TSK or Mamdani types) are tuned by a GA. Two other approaches are: [8], which employs linguistic hedge operators, selected by a GA, to tune membership functions, and [10] where a hierarchical self-organized GAbased scheme is proposed.

Recently, most works that make use of GA to tune FLCs focus on real applications [19, 21, 22]. Type-2 FLCs have also been tuned through GA [18]. Additionally, some non-GBMH works for tuning an FLC have also considered Particle Swarm Optimization [23] and other bio-inspired algorithms [24].

Few attempts, however, have been made to build an FLC by using GP, despite its dynamic structure that benefits rule base codification [6]. The first works in this sense were [25] and [26], which used a type-constrained GP to build a fuzzy rule based system. In [27] an FLC based on GP for mobile robot path tracking is presented. More recently, [12] proposes the use of a GP variant to build a TSK FLC. All those approaches adapt the GP structure to formulate an FLC in a canonical way, similarly to a GA common procedure. Some intrinsic advantages of GP are effectively used by these authors, but many possibilities arise, such as the use of combinations of different t-norms and $t$ conorms, of linguistic hedges and of different aggregation operators.

All approaches previously discussed focus on Pittsburgh-type GFC, i.e., an individual of the population encodes a whole fuzzy rule set $[3,6]$. Then, methods that consider an individual as a fuzzy ruleMichigan, Genetic Cooperative-Competitive Learning and Iterative Rule Learning - have not been noticed in the literature [17].

GPFIS-Control is a novel GFC based on MultiGene Genetic Programming. This model builds a Pittsburgh-type Fuzzy Rule Based System, making use of a different reasoning method to learn fuzzy rules.

\section{Multi-Gene Genetic Program- ming}

Genetic Programming (GP) [28-29] belongs to the Evolutionary Computation field. Typically, it employs a population of individuals, each of them denoted by a tree structure that codifies a mathematical equation, which describes the relationship between the output $Y$ and a set of input terminals $X_{j}(j=1, \ldots, J)$ (features, in the current work).

Multi-Gene Genetic Programming (MGGP) [15-16] denotes an individual as a structure of trees, also called genes, that receives $X_{j}$ and tries to predict $Y$ (Figure 1). Each individual is composed of $D$ functions $f_{d}(d=1, \ldots D)$ that map $X_{j}$ variables to $Y$ through user-defined mathematical operations. In GP terminology, the $X_{j}$ input variables are included in the Terminal set, while the mathematical operations (plus, minus, etc.) are inserted in the Function Set (or Mathematical Operations Set).

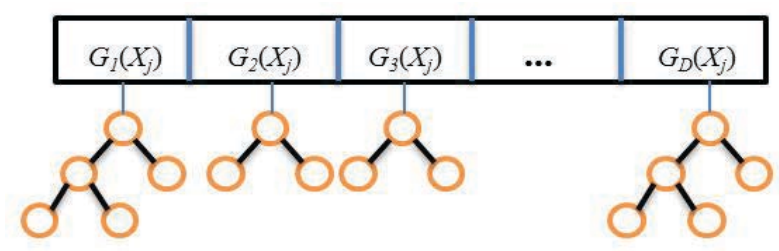

Figure 1. Example of a multi-gene individual

With respect to genetic operators, mutation in MGGP is similar to that in GP. As for crossover, the level at which the operation is performed must be 
specified: it is possible to apply crossover at high and low levels. The low level is the space where it is possible to manipulate the structures (terminals and functions) of equations present in an individual. The high level, on the other hand, is the space where expressions can be manipulated in a macro way. In this case, mutation and low level crossover operations are similar to those performed in GP. Figure 2 presents a multi-gene individual with five equations $(D=5)$. Figure 2 a shows the mutation operation, while Figure $2 \mathrm{~b}$ a low level crossover.

An example of high level crossover is displayed in Figure 2c. By observing the dashed lines, it can be seen that the equations were switched from an individual to the other. The cutting point can be symmetric - the same number of equations is exchanged between individuals -, or asymmetric. Intuitively, high level crossover has a deeper effect on the output than low level crossover or mutation has. In of the proposed GPFIS-Control model, the asymmetric high level crossover is considered.

In general, the evolutionary process in MGGP differs from GP due to the addition of two parameters: maximum number of trees per individual and high level crossover rate. For the first parameter, a high value is always used in order to avoid creating obstacles in the evolutionary process (i.e., not allowing the MGGP individual to create more trees, which could be necessary to provide solutions for complex problems). On the other hand, the high level crossover rate, similar to other genetic operators' rates, needs to be adjusted and its value is always determined by performing different tests.

\section{GPFIS-Control}

The GPFIS-Control model is shown in Figure 3. The control signal $y_{t}$ is sent to the plant at time $t$ $(t=0,1, \ldots, T)$. The plant outputs $z_{t k}(k=1, \ldots K)$ are compared with the setpoint value, so that the result of the difference between each plant's output and its respective setpoint (the error signal $x_{t k}=z_{t k}-\operatorname{Ref}_{k}$ ) is presented to the GPFIS-Control model. By using $x_{t k}$ it is possible to build a control signal $y_{t}$ to satisfy a performance criteria (Fitness function $\mathrm{g}\left(x_{t k}, t\right)$ ).

The GPFIS-Control model is comprised of four modules: fuzzification, inference, defuzzification and evaluation. The inference process begins when each feedback error $x_{t k}$ is mapped on fuzzy sets. Then, functions that map each linguistic state of $x_{t k}$ to a state of $y_{t}$ are synthesized based on MGGP principles. The crisp control signal is obtained through the defuzzification process. This solution is evaluated and then selection and recombination operators are applied. These steps are repeated until a stopping criterion is met. These four modules are described in details in the following sub-sections.

\subsection{Fuzzification}

Let $x_{t k}$ and $y_{t}$ admit $J$ distinct linguistic terms, or fuzzy sets $(j=1, \ldots, J)$. These are defined by normalized and uniformly distributed membership functions [30]. Figure 4 presents an example of the fuzzification of the $k$-th plant output, with seven membership functions with the following linguistic terms: NB - Negative Big; NM - Negative Medium; NS - Negative Small; NZ - Near Zero; PS - Positive Small; PM - Positive Medium; and PB - Positive Big (in this case, $j=1,2, \ldots, 7$ ).

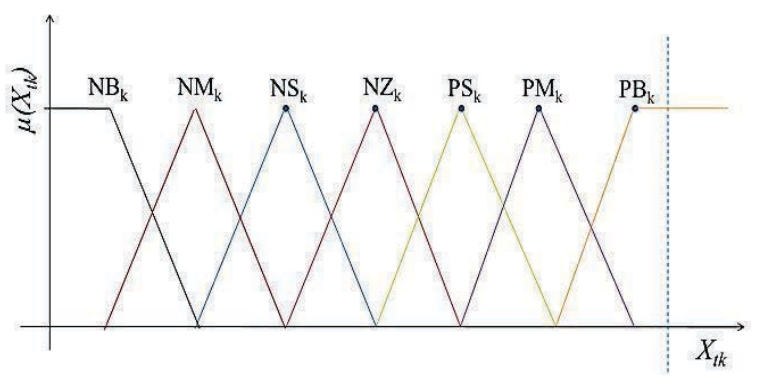

Figure 4. Example of membership functions

After fuzzification of each input $x_{t k}$, the GPFISControl inference process initiates.

\subsection{Fuzzy Inference}

The inference procedure consists of three stages: Formulation, Partitioning and Aggregation. In Formulation stage, t-norm, t-conorm, linguistic hedges and negation operators are defined. In Partitioning stage, the mechanism that connects each antecedent with a consequent is established. Finally, in Aggregation stage, operators used to combine all rules are defined.

\subsubsection{Formulation}

Through each ${ }_{A j k}\left(x_{t k}\right)$ (membership degree of $x_{t k}$ to a fuzzy set $A_{j k}$ ), GPFIS-Control evolves a 
(a)

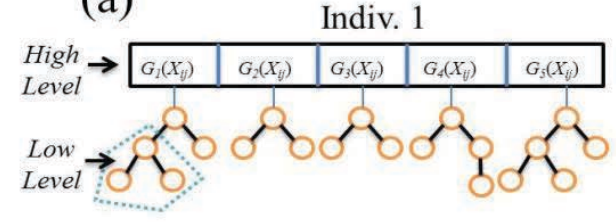

(b)

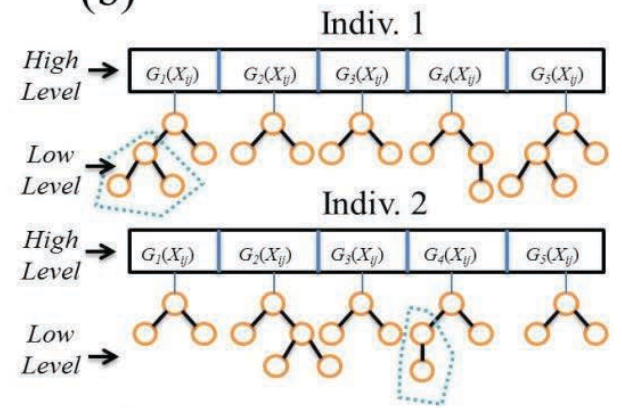

(c)

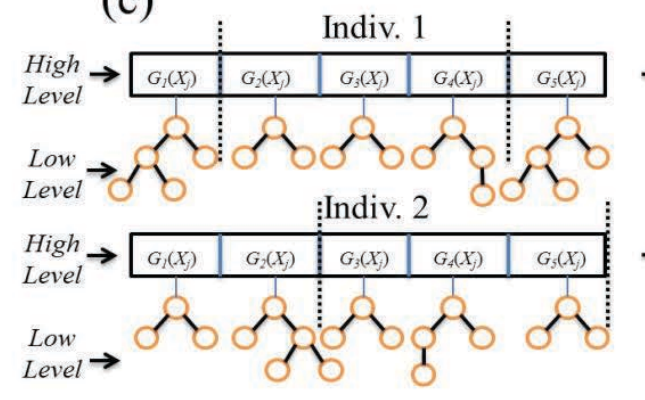

Indiv. 1

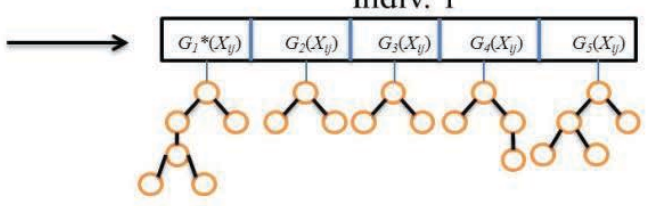

Indiv. 1
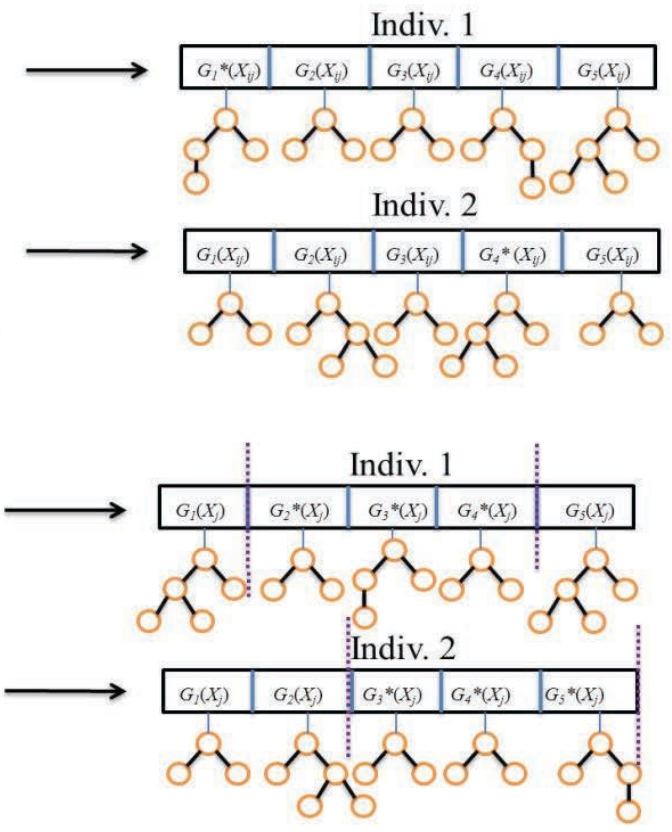

Figure 2. Example of Multi-Gene Genetic Programming recombination operators

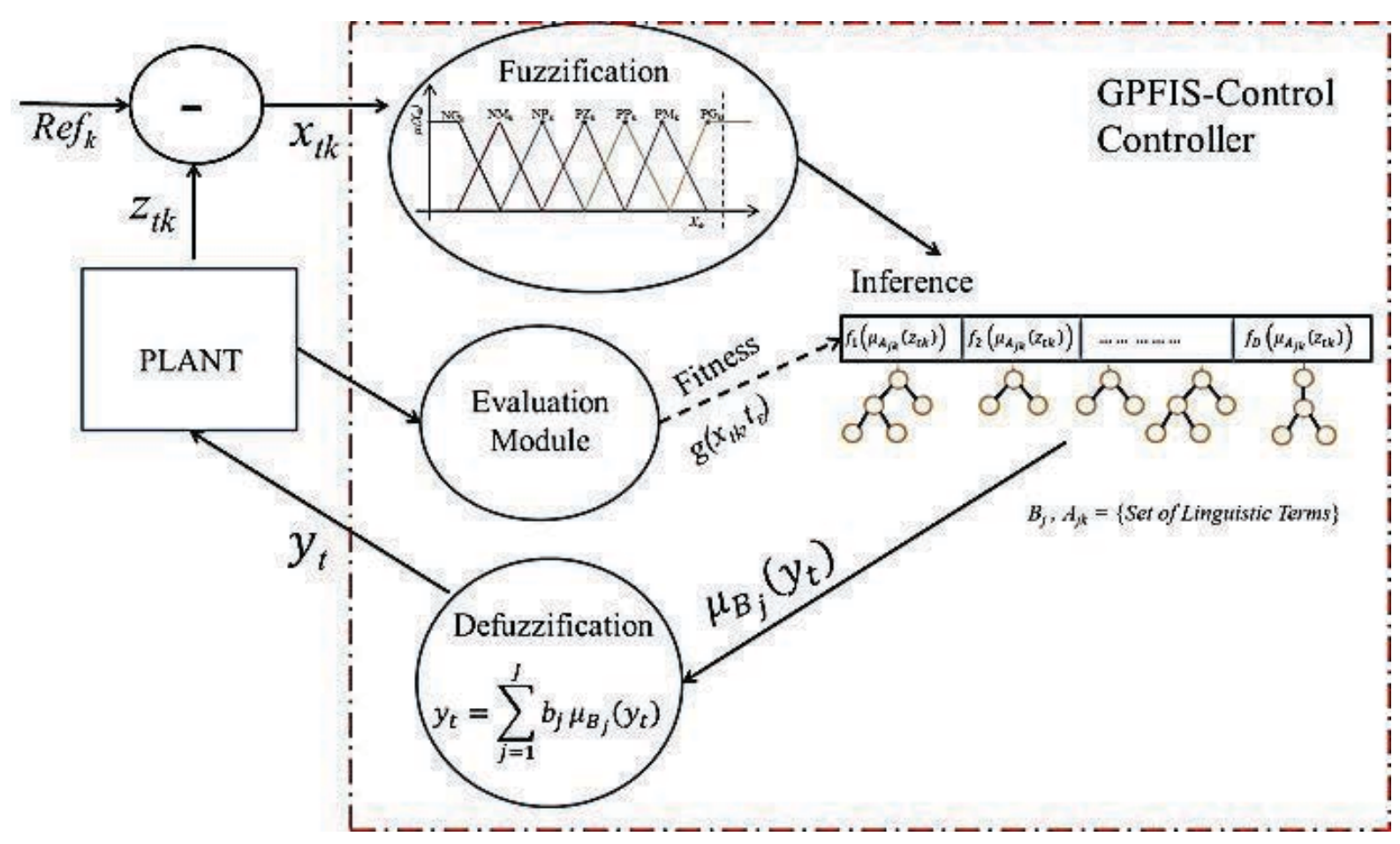

Figure 3. Block diagram of GPFIS-Control model 
controller whose ouput has several terms $\left(B_{1}=\right.$ Negative Big, ..., $B_{7}=$ Positive Big, for example), with membership degrees given by:

$$
\begin{aligned}
& \mu_{B_{1}}\left(y_{t}\right)=g\left[f_{d \in s_{1}}\left(\mu_{A_{j 1}}\left(x_{t 1}\right), \ldots, \mu_{A_{j K}}\left(x_{t K}\right)\right)\right] \\
& \mu_{B_{2}}\left(y_{t}\right)=g\left[f_{d \in s_{2}}\left(\mu_{A_{j 1}}\left(x_{t 1}\right), \ldots, \mu_{A_{j K}}\left(x_{t K}\right)\right)\right] \\
& \ldots \\
& \mu_{B_{J}}\left(y_{t}\right)=g\left[f_{d \in s_{J}}\left(\mu_{A_{j 1}}\left(x_{t 1}\right), \ldots, \mu_{A_{j K}}\left(x_{t K}\right)\right)\right]
\end{aligned}
$$

where $f_{d \in s_{j}}\left(\mu_{A_{j 1}}\left(x_{t 1}\right), \ldots, \mu_{A_{j K}}\left(x_{t K}\right)\right)$ represents a set of functions, where each one combines all $\mu_{A_{j k}}\left(x_{t k}\right), k=1, . ., K$, by using a set of user-defined mathematical operations; $s_{j}(j=1, \ldots, J)$ is an index set that describes which $d$-th function $f_{d}$ is related to the $j$-th consequent term $\left(d \in s_{j}\right)$. Methods to define $s_{j}$ are best described in the Partitioning stage. In order to each function $f_{d}$ associated to $s_{j}$ behave as a fuzzy rule, it needs to employ t-norm, t-conorm, negation and linguistic hedges operators, with the aim to represent logic connectives for each linguistic term induced by $\mu_{A_{j k}}\left(x_{t k}\right)$. Finally, $g$ aggregates the activation degrees of each rule set (represented by $f_{d \in s_{j}}$ ) in a final value. Therefore, if a set $A_{j k}$ is activated, GPFIS-Control builds a rule set (function set) that combines all membership degrees $\left(\mu_{A_{j k}}\left(x_{t k}\right)\right)$ and produces an action.

In Formulation stage, some parameters of GPFIS-Control must be defined. In MGGP, initial parameters are called Terminals (input variables) and Mathematical Operations or Function Set (plus, times, etc.). In GPFIS-Control, on the other hand, the terminology will be Input Fuzzy Sets and Fuzzy Operators Set, respectively. Table 1 presents the initial user-defined parameters.

Table 1. Input Fuzzy Sets and Fuzzy Operators

\begin{tabular}{|l|l|}
\hline Input Fuzzy Sets & $\begin{array}{l}\text { Fuzzy Operators } \\
\text { Set }\end{array}$ \\
\hline$\mu_{A_{j 1}}\left(x_{t 1}\right), \ldots, \mu_{A_{j K}}\left(x_{t K}\right)$ & $\begin{array}{l}\text { t-norms, t-conorms, } \\
\text { negation and lin- } \\
\text { guistic hedges oper- } \\
\text { ators }\end{array}$ \\
\hline
\end{tabular}

Subsequently, by using the Fuzzy Operators Set, the $\mu_{A_{j k}}\left(x_{t k}\right)$ is combined in order to best describe the actions $\mu_{B_{j}}\left(y_{t}\right)$ taken by the controller. It is possible to enter a negated or modified ("hedged") fuzzy set in the Input Fuzzy Sets stage, instead of using negation and linguistic hedge operators in the Fuzzy Operators Set stage. This entails a larger search space, but can be of help in rules analysis. In this case, this procedure has been used to make the fuzzy rules simpler.

By using the operators and membership functions shown in Table 1, the MGGP builds premises of fuzzy rules as follows:

$$
\text { "If } X_{1} \text { is } A_{j 1} \text { and .... and } X_{K} \text { is } A_{j K} \text { " }
$$

where negation and linguistic hedges can operate on each element of the antecedent term.

\subsubsection{Partitioning}

Let $S=\left\{s_{1}, s_{2}, \ldots, s_{J}\right\}$ be the set of indicators $s_{j}$, where each $s_{j}$ represents which $f_{d}(d=\{1, \ldots, D\})$ is related to the $j$-th consequent $B_{j}$. The method that describes which $d$-th function is associated to $s_{j}$ is called Uniform Division. This partitioning method makes use of a simple heuristic, given by:

1 Compute: $U=\left\lfloor\frac{D}{J}\right\rfloor$ (where $\lfloor$.$\rfloor is the floor oper-$ ator).

2 Partition: $s_{1}=\{1, \ldots, U\}, s_{2}=\{U+1, \ldots, 2 * U\}, \ldots$, $s_{J}=\left\{U^{*}(J-1)+1, \ldots, U^{*} J\right\}$.

As an example, consider $D$ (number of functions) $=10$ and $J$ (number of consequent terms) $=$ 5. Thus $U=2, s_{1}=\{1,2\}, s_{2}=\{3,4\}, s_{3}=\{5,6\}, s_{4}=$ $\{7,8\}, s_{5}=\{9,10\}$. Figure 5 illustrates this process.

In summary, each $f_{d}$ is uniformly divided for each $s_{j}$ so that a consequent has at least one rule associated to it. This method is similar to others GFS based on GP, such that consequent and antecedent terms are both synthesized. Through the definition of the rule set associated to each consequent $\left(S=\left\{s_{0}, s_{1}, s_{2}, \ldots, s_{J}\right\}\right)$, the next step is to aggregate them, in order to generate a final degree of activation.

\subsubsection{Aggregation}

Many different aggregation operators may be found in the literature [31-32]. 


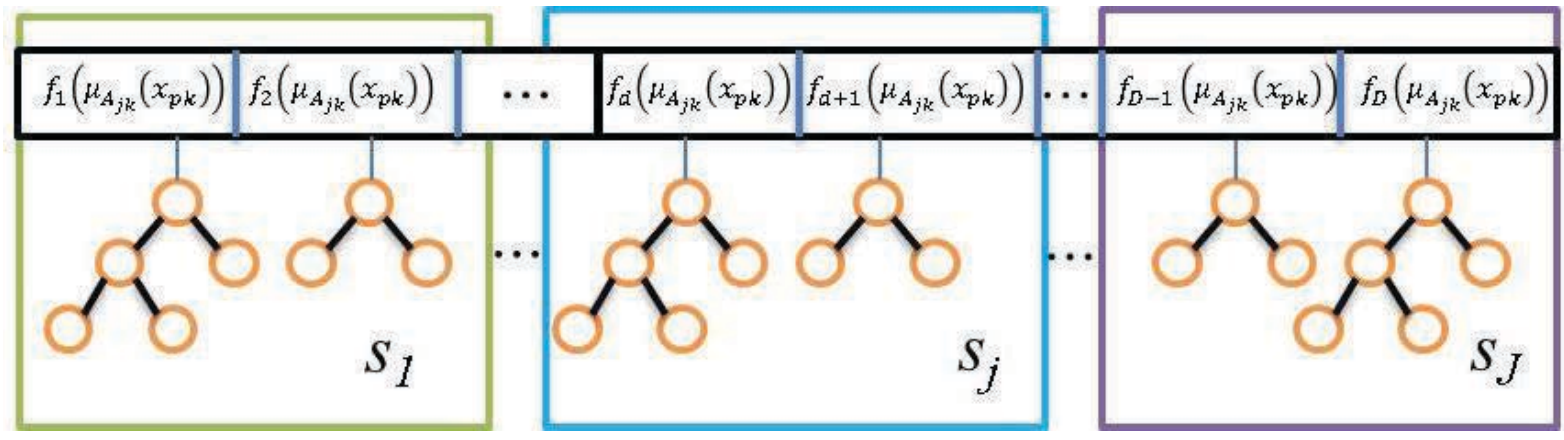

Figure 5. Uniform division procedure

Some examples of $g\left[f_{d \in s_{1}}\left(\mu_{A_{j 1}}\left(x_{t 1}\right), \ldots, \mu_{A_{j K}}\left(x_{t K}\right)\right)\right]$ are:

$-g \rightarrow \max \left[f_{d \in s_{1}}\left(\mu_{A_{j 1}}\left(x_{t 1}\right), \ldots, \mu_{A_{j K}}\left(x_{t K}\right)\right)\right] \quad:$ max aggregation operator are the most common used on Mamdani type FIS.

$-g \rightarrow \frac{\sum-\left\{\mathrm{d} \in \mathrm{s}_{\mathrm{j}}\right\}}{\operatorname{card}\left(s_{j}\right)}\left[\left[f_{d \in s_{1}}\left(\mu_{A_{j 1}}\left(x_{t 1}\right), \ldots, \mu_{A_{j K}}\left(x_{t K}\right)\right)\right]:\right.$ arithmetic mean operator intends to provide equal weights for each element of the rule set associated to the $\mathrm{j}$-th consequent.

In [31], several aggregation operators are presented. It can be shown that t-norms and t-conorms are special cases of aggregation operators. In the experiments both the arithmetic mean and maximum operators have been used. Once the aggregation operators have been defined, it is possible to compute the membership degrees for different actions $\mu_{B_{j}}\left(y_{t}\right)$ taken by the controller. The defuzzified control signal $y_{t}$ is then computed.

\subsection{Defuzzification}

Basically, a defuzzification method (center of gravity, mean of maximum, etc.) produces a crisp value that is an interpretation of the information contained in the output fuzzy set resultant from the inference process. In GPFIS-Control the height method is used:

$$
y_{t}=\frac{\sum_{j=1}^{J} b_{j} \mu_{B_{j}}\left(y_{t}\right)}{\sum_{j=1}^{J} \mu_{B_{j}}\left(y_{t}\right)}
$$

where $b_{j}$ represents the center (location) parameter of each $B_{j}$. The maximum height method may be employed when the control signal assumes values in some finite set:

$$
y_{t}=\frac{\sum_{j=1}^{J} \phi_{j} b_{j} \mu_{B j}\left(y_{t}\right)}{\sum_{j=1}^{J} p h i_{j} \mu_{B j}\left(y_{t}\right)}
$$

where $\phi_{j}$ is an indicator function, such that $\phi_{j}=1$, when $\mu_{B_{j}}\left(Y_{t}\right)>\mu_{B_{l}}\left(Y_{t}\right)$, for all $l=1, \ldots, J$, e $l \neq j$, e $\phi_{j}=0$, otherwise.

Figure 6 presents an illustration of the difference between these procedures. We obtained: $\mu_{N M}\left(Y_{t}\right)=0,8, \mu_{P Z}\left(Y_{t}\right)=0,6$, while $\mu_{N G}\left(Y_{t}\right)=$ $\mu_{N P}\left(Y_{t}\right)=\mu_{P P}\left(Y_{t}\right)=\mu_{P M}\left(Y_{t}\right)=\mu_{P G}\left(Y_{t}\right)=0$.

Then, according to Eq. (4):

$$
Y_{t}=\frac{-20 \cdot 0,8+0 \cdot 0,6}{0,8+0,6}=-11,43
$$

If it was used maximum height method, the response would be -20 , because the presence of only one maxima value. Height method provides a smoother transition between responses than maximum height.

\subsection{Evaluation}

The right definition of the fitness function is crucial for obtaining a good performance of the GPFIS-Control model. For optimal tracking of a trajectory, a possible fitness function is the Mean Squared Error (MSE):

$$
M S E=\frac{1}{K} \sum_{k=1}^{K}\left(x_{t k}\right)^{2}
$$

When the MSE is minimized, the GPFISControl model successfully obtains a trajectory close to the setpoint. In minimum time problems, the fitness function may be the time $(t)$ the output takes to reach an $\operatorname{MSE}<\varepsilon$, where $\varepsilon$ is a tolerance. 
GPFIS-Control tries to reduce the size and complexity of the rule base by employing a simple heuristic called Lexicographic Parsimony Pressure [33]. This technique is only used in the selection phase: given two individuals with the same fitness, the best one is that with fewer nodes. Fewer nodes indicate rules with fewer antecedents, hedge and negation operators, as well as few functions $\left(f_{d}\right)$, and, therefore, a smaller rule set.

After the evaluation procedure, a set of individuals are selected (using tournament procedure) and recombined. Then, in a subset of the population, the mutation (Figure 7a), low-level crossover (Figure 7b) or high-level crossover (Figure 7c) operators are applied. Finally, a new population is generated.

This process is repeated until a stopping criteria is met. At this moment, the final population is returned.

\section{Case Studies}

Two benchmark problems have been considered to evaluate the GPFIS-Control model: cartcentering $[25,28]$ and inverted pendulum $[9,12]$. The cart-centering problem has been used to assess the performance of GPFIS-Control in comparison with other GFCs. The application to the inverted pendulum made use of the GPFIS-Control parameters obtained in the tuning for the cart-centering problem. Results were compared to those presented in [12].

\subsection{Experimental Settings}

\subsubsection{Cart-centering Problem}

The cart-centering problem consists of a cart with mass $m$ moving on a frictionless rail; at some instant $t$ its position is $x_{t}(m)$, with velocity $v_{t}(\mathrm{~m} / \mathrm{s})$. The cart must stop $\left(v_{t}=0\right)$ at a user-defined setpoint ref. Tolerance values $\varepsilon$ may be considered, so that $\left|x_{t}-r e f.\right|<\varepsilon$ and $\left|v_{t}-r e f.\right|<\varepsilon$. The plant dynamics is given by Equations (7) and (8):

$$
\begin{gathered}
v_{t+\tau}=v_{t}+\tau \frac{F_{t}}{m} \\
x_{t+\tau}=x_{t}+\tau v_{t}
\end{gathered}
$$

where $\tau$ is the sampling period and $F_{t}$ is the force $(N)$ applied by the controller to the cart at time $t$. The objective is to reach the setpoint in minimum time. The performance of GPFIS-Control has been compared to the GFC presented in [25]. Several configurations for GPFIS-Control (t-norms, aggregation operators, etc.) have been evaluated. To perform a fair comparison, configurations were the same as those in [25] for all variables and parameters (Table 2 displays these values).

GPFIS-Control is required to move the cart until $\left|x_{t}-0\right|<0.5$ and $\left|v_{t}-0\right|<0.5$, given 16 initial values uniformly distributed on the $x_{t}$ domain. The fitness function has been defined as:

$$
\text { Fitness }=t_{\varepsilon}+\sum_{t}\left|x_{t}\right|
$$

where $t_{\varepsilon}$ is the time needed to satisfy the stopping criteria $\left(\left|x_{t}-0\right|<0.5\right.$ and $\left.\left|v_{t}-0\right|<0.5\right)$. An individual in the GPFIS-Control population is considered unfeasible if it cannot stop the cart in 10 seconds (500 sampling steps).

Table 2. Configurations Set for Cart-Centering Problem

\begin{tabular}{|l|l|}
\hline Variable & Domain \\
\hline$F_{t}$ & {$[-2.5,2.5] \mathrm{N}$} \\
\hline$v_{t}$ & {$[-2.5,2.5] \mathrm{m} / \mathrm{s}$} \\
\hline$x_{t}$ & {$[-2.5,2.5] \mathrm{m}$} \\
\hline Parameter & Value \\
\hline$\tau$ & $0.02 \mathrm{~s}$ \\
\hline$\varepsilon$ & 0.5 \\
\hline$m$ & $2.0 \mathrm{~kg}$ \\
\hline ref. & $x_{t}=v_{t}=0$ \\
\hline
\end{tabular}

After the best solution is found, it is applied to 1000 initial random positions in order to evaluate the time taken by GPFIS-Control to stop the cart. In order to perform a fair comparison with [25], the following procedure has been executed 10 times: $(i)$ generate a GPFIS-Control model and, (ii) apply it on 1000 random position, in order to produce statistical relevant results. The best GPFIS-Control model is obtained, in each execution, after 25000 evaluations (population size $=50$ and number of generations $=500$, respectively). Table 3 displays all the parameters of the GPFIS-Control model. 


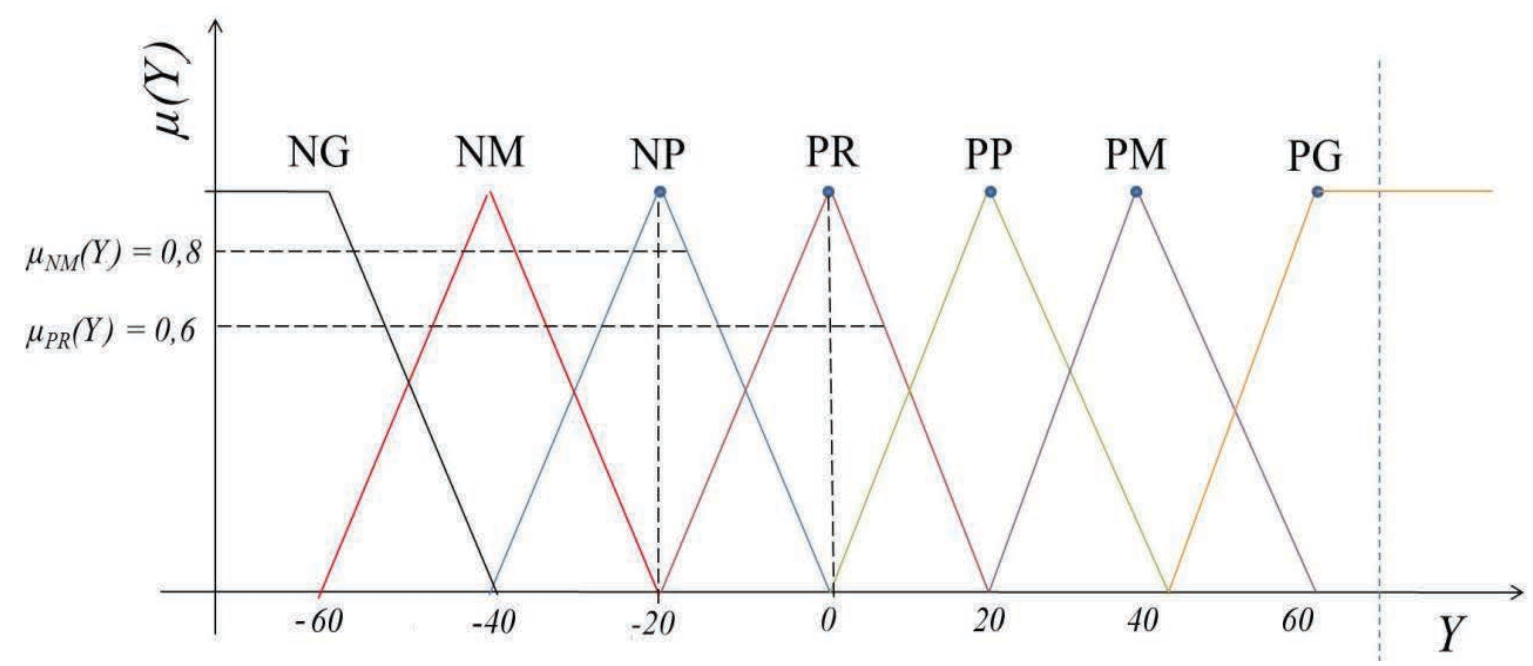

Figure 6. Example for defuzzification procedures

Table 3. Remaining GPFIS-Control parameters

\begin{tabular}{|l|l|}
\hline Parameter & Value \\
\hline Tournament Size & 5 \\
\hline Maximum Tree Depth & 5 \\
\hline Elitism Rate & $1 \%$ \\
\hline $\begin{array}{l}\text { Maximum rules per in- } \\
\text { dividual }\end{array}$ & 50 \\
\hline $\begin{array}{l}\text { Low level crossover } \\
\text { rate }\end{array}$ & $75 \%$ \\
\hline $\begin{array}{l}\text { High level crossover } \\
\text { rate }\end{array}$ & $50 \%$ \\
\hline Mutation rate & $20 \%$ \\
\hline $\begin{array}{l}\text { Direct reproduction } \\
\text { rate }\end{array}$ & $5 \%$ \\
\hline $\begin{array}{l}\text { Input Fuzzy Sets } \\
\text { cal Negation* of each } \\
\text { Fuzzy Set per variable }\end{array}$ \\
\hline Fuzzy Operators Set & $\begin{array}{l}\text { t-norm: product }, \\
\text { others: described for } \\
\text { each experiment }\end{array}$ \\
\hline
\end{tabular}

* used only when told

\subsubsection{Inverted Pendulum Problem}

The second experiment consisted of an application of GPFIS-Control to the inverted pendulum problem. Results were then compared to those of [12]. In this problem, a cart of mass $M$ with a pole of mass $m$ and height $\lambda$ attached to its center moves on a frictionless rail. The controller must apply $F_{t}$ in order to increase or decrease $v_{t}$ and consequently change the angular velocity $\omega_{t}$ and the pendulum angle $\theta_{t}$. The dynamic model $[12,28]$ is described below:

$$
\begin{gathered}
\phi_{t}=\frac{g \sin \theta_{t}+\cos \left(\theta_{t}\right) \Psi}{\lambda\left[\frac{4}{3}-\frac{m \cos ^{2} \theta_{t}}{M+m}\right]} \\
\psi=\frac{-F_{t}-m \lambda \omega_{t} \theta_{t}^{2} \sin \theta_{t}}{M+m} \\
\omega_{t+1}=\omega_{t}+\tau \phi_{t} \\
\theta_{t+1}=\theta_{t}+\tau \omega_{t} \\
a_{t}=\frac{F_{t}+m \lambda\left[\theta_{t}^{2} \sin \theta_{t}-\omega_{t} \cos \theta_{t}\right]}{M+m} \\
v_{t+1}=v_{t}+\tau a_{t}
\end{gathered}
$$

where $\phi_{t}$ is the angular acceleration and $\tau$ is the sampling step. In order to perform a fair comparison with [12], the feasible domain for each 
(a)

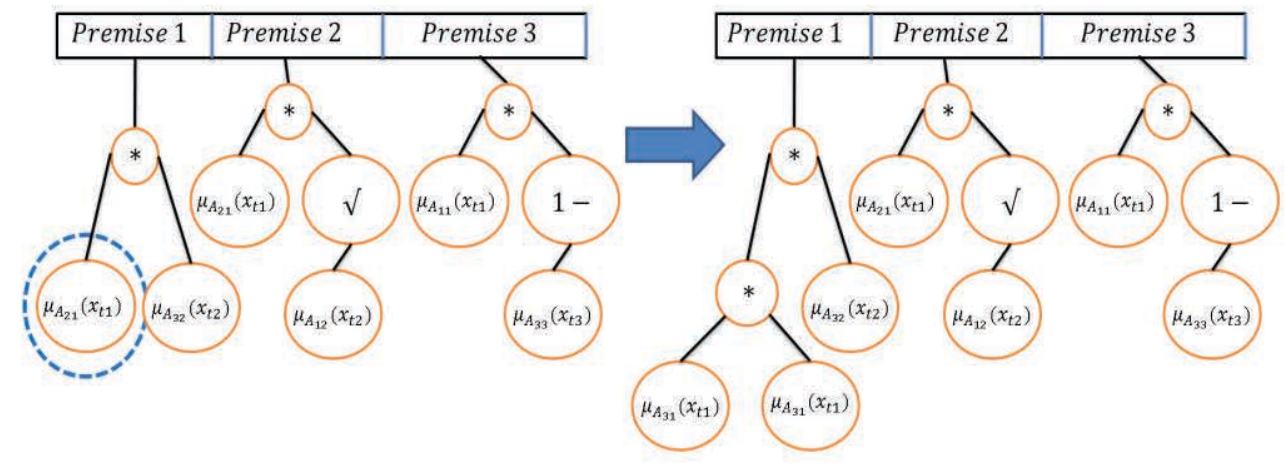

(b)
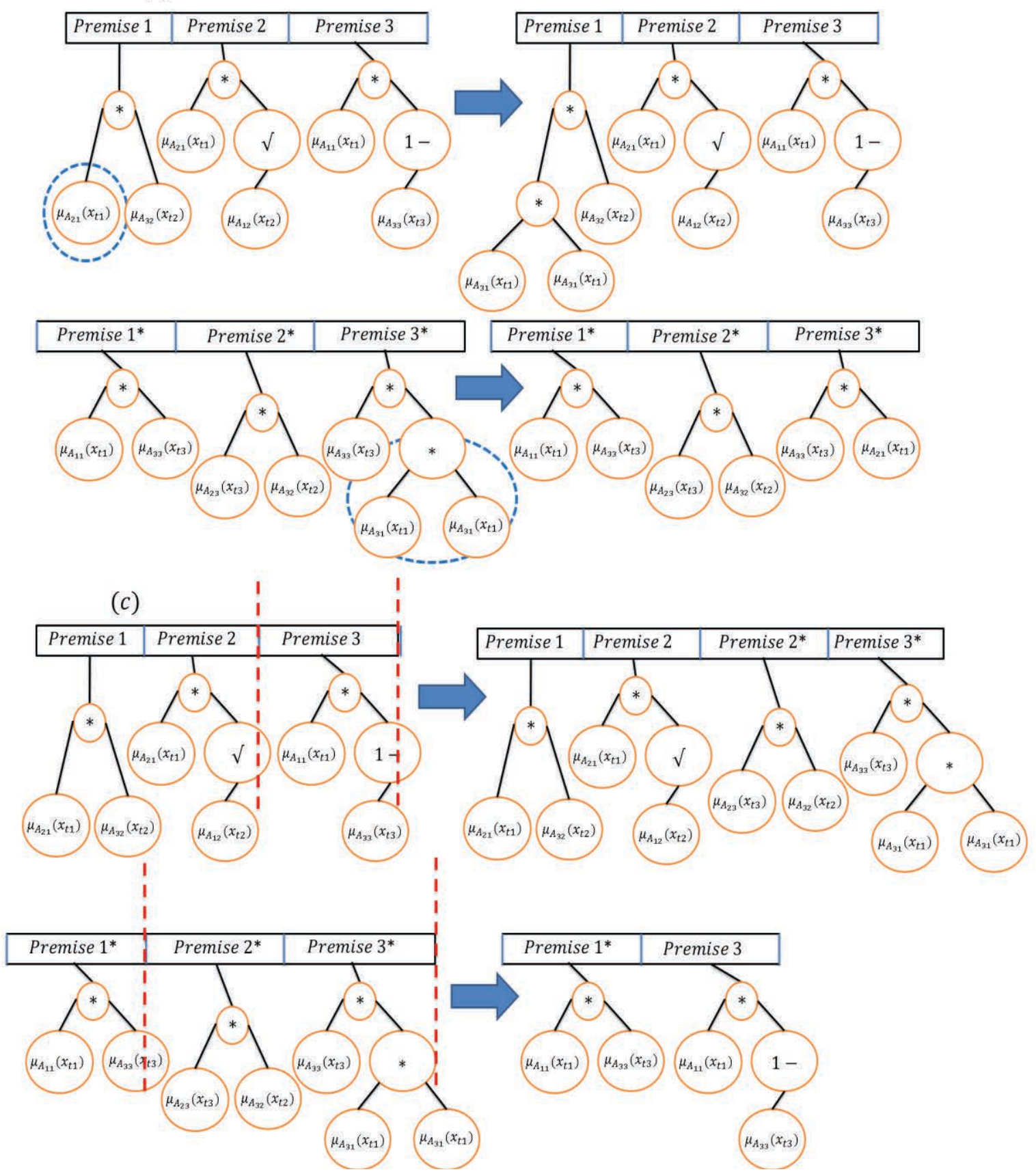

Figure 7. Example of recombination operators applied in GPFIS-Control solutions 
variable was set as: $\omega_{t} \in[-0.87,0.87] \mathrm{rad} / \mathrm{s}$, $\theta_{t} \in[-0.34,0.34] \mathrm{rad}, F_{t} \in[-25,25] N$, while $x_{t}$ and $v_{t}$ are unconstrained, $M=1 \mathrm{~kg}, m=0.1 \mathrm{~kg}, \lambda$ $=0.5 \mathrm{~m}, g=9.8 \mathrm{~m} / \mathrm{s}^{2}$ and $\tau=0,01 \mathrm{~s}$. Two initial conditions were considered: $\theta_{0}=\{-0,18,0,18\} \mathrm{rad}$, with $\omega_{0}=\{0,0\} \mathrm{rad} / \mathrm{s}$ and the setpoint is $\mathrm{ref}=0 \mathrm{rad}$ with $\varepsilon=0.01$. The time allowed for the position $\left|\theta_{t}-0\right|<$ 0.01 to be reached is at most 1 second (100 sampling steps).

As in [12], 100,000 evaluations (population size $=100$ and number of generations 1000) have been made. All this procedure was repeated 10 times, in order to generate statistical relevant results. Table 3 exhibits the remaining parameters used. The fitness function is [12]:

$$
\text { Fitness }=\sum_{t=1}^{100}\left(\theta_{t}-r e f\right)^{2}
$$

In both experiments seven fuzzy sets have been assigned to each variable $\left(F_{t}, x_{t}, v_{t}, \omega_{t}, \theta_{t}\right)$, as shown in Figure 4. In some cases, the negation of a fuzzy set was entered in the Input Fuzzy Sets stage of the GPFIS-Control routine (as described in section 4.2.1). All experiments were performed in MATLAB R2010a [34].

\subsection{Results and Discussions}

\subsubsection{Cart-Centering Problem}

The main results obtained with the cartcentering problem are presented in Table 4. GPFISControl was tested with the linguistic hedge square root, the classic negation operator, different aggregation operators (max and average) and different defuzzification methods (height and maximum height). It can be seen that for almost all configurations, the use of the average aggregation operator reduces by about $39 \%$ the mean time taken by the controller to position the cart at $\left|x_{t}-0\right|<0.5$ and $\mid v_{t}$ $-0 \mid<0.5$. It may also be noted that the maximum height defuzzification reduces that time in $14 \%$ in average. However, the use of the negation operator does not incur in any substantial time decrease, although fewer rules are generated. In fact, the negation operator has a summarizing power, due to the enlargement of a fuzzy set support in the universe of discourse.
The best configurations were obtained with the following parameters: maximum height method for defuzzification and average as the aggregation operator. Figure 8 present the 16 initial and final positions when $\left|x_{t}-0\right|<0.5$ and $\left|v_{t}-0\right|<0.5$. Figure 9 exhibits the response surface for GPFISControl best configuration for (a): maximum height defuzzification method and (b): height defuzzification method. It can be seen that the surface for (b) is smoother than that for (a), due to a broader set of values that $F_{t}$ can assume when the height method is chosen.

The average best result for GPFIS-Control (135.8 steps) compares favorably with those of [25] (158 steps) and [35] (149 steps). The optimal solution is 129 steps.

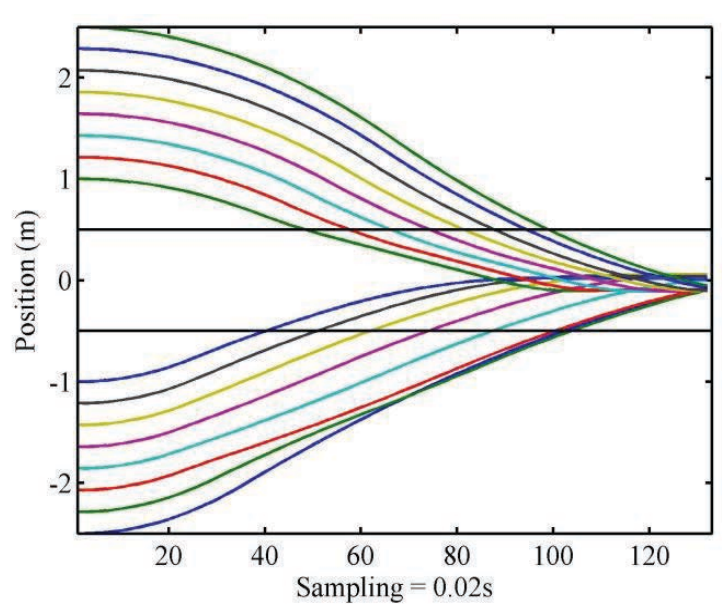

Figure 8. Initial and final position for the best individual in an execution of GPFIS-Control, using Product+Root-Sq+MaxHeight and average aggregation operator

\subsubsection{Inverted Pendulum}

Based on the best configuration previously established (Product + Root-Sq + Average + MaxHeight), GPFIS-Control has been applied to the inverted pendulum problem. Figure 10 shows the controller's behavior, generated by the best individual in 100,000 evaluations, given two initial conditions: $\theta_{0}=\{-0,18,0,18\} \mathrm{rad}$, with $\omega_{0}=\{0,0\} \mathrm{rad} / \mathrm{s}$. The average best result found for GPFIS-Control was 0.27 seconds to reach and stay at $\left|\theta_{t}-0\right|<0.01$ during 1.00 second, generating 14 rules in average. In [12] the GFC took 0.61 seconds to perform the same task, however producing fewer rules ( 7 rules). 

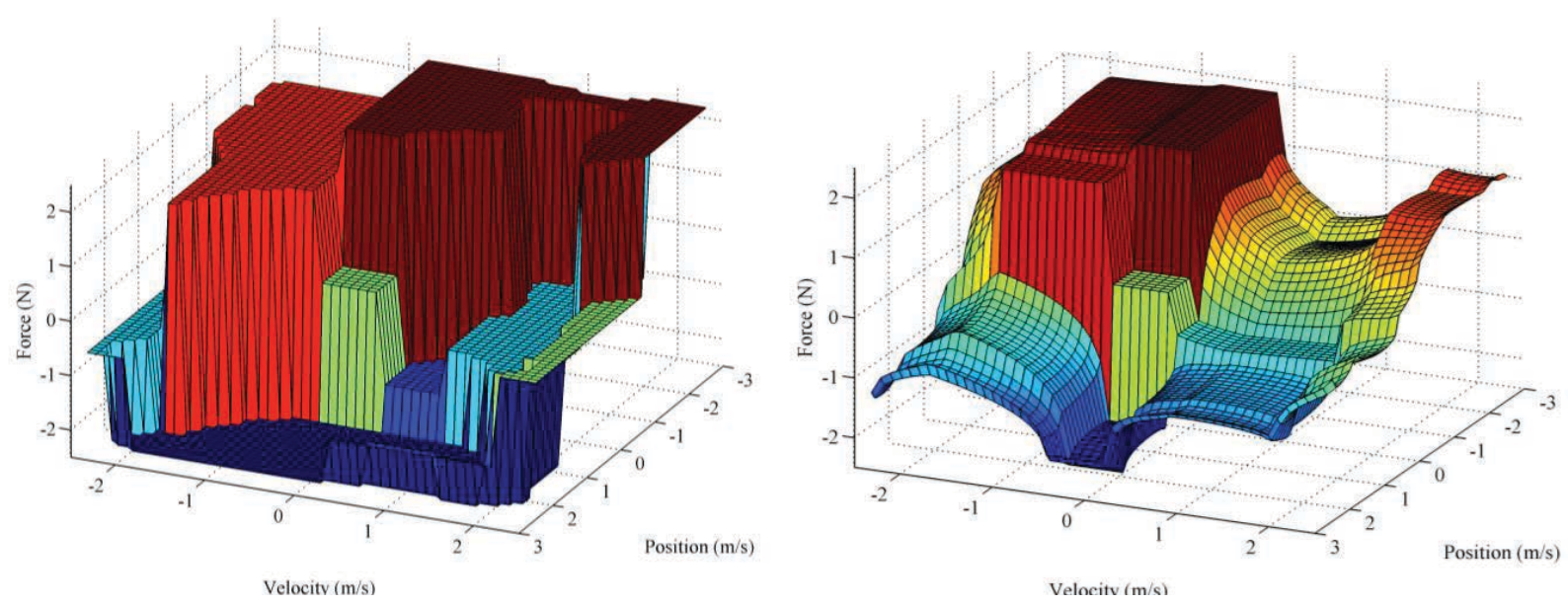

Figure 9. Response surface for the best individual in cart-centering for different defuzzification methods: (a): maximum height; (b) height

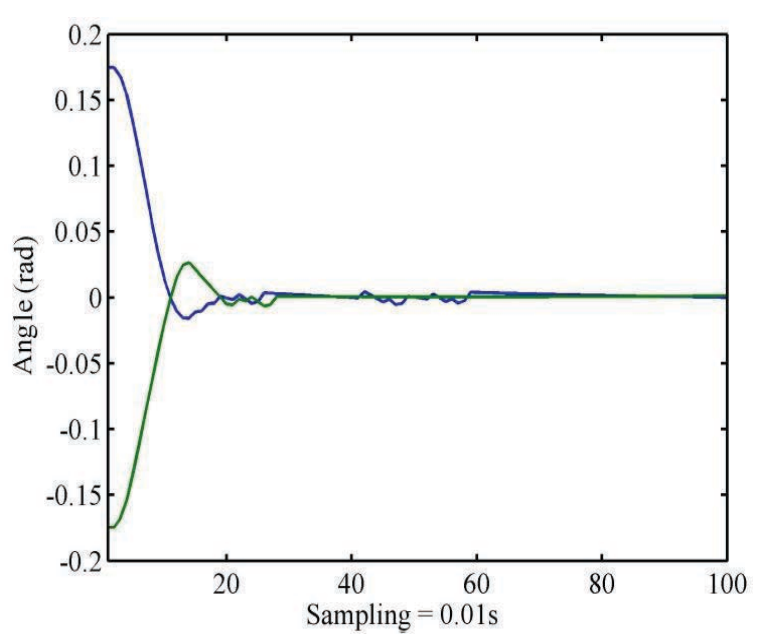

Figure 10. Initial and final position for the best individual in an execution of GPFIS-Control, using

Product+Root-Sq+MaxHeight and average aggregation operator configurations

\section{Conclusion}

A novel approach for solving control problems has been presented. It consists of a Genetic Programming Fuzzy Inference System for Control tasks (GPFIS-Control), based on Multi-Gene Genetic Programming. The proposed GPFIS-Control model considers the usual stages of a Genetic Fuzzy Inference System: fuzzification, inference, defuzzification and evaluation.

The performance of GPFIS-Control has been evaluated through two benchmarks problems: cartcentering and inverted pendulum. The use of dif- ferent aggregation, defuzzification and negation operators has been analyzed. It was shown that the right choice of defuzzification and aggregation operators improves results, while the use of negation may reduce the number of rules. When compared to other Genetic Fuzzy Controllers, GFPIS-Control has shown a better performance in average.

Future works shall consider other benchmark and real world problems, as well as new methods in formulation, partitioning and aggregation. For example, rules could be aggregated by using a weighted average, with adaptive weights for the rules during the controller operation. This could improve results with fewer rules. The use of others partitioning methods can also improve the performance, helping GPFIS-Control model to select the most promising rules for each consequent. A sensitivity analysis of some parameters (tournament size, maximum tree depth, etc.) would help to evaluate their influence on the final result.

\section{References}

[1] J. M. Mendel, Fuzzy logic systems for engineering: a tutorial, Proceedings of the IEEE, Vol.83, No.3, 1995, p.345-377.

[2] C. Elmas, C., O. Deperlioglu, and H. H. Sayan, Adaptive fuzzy logic controller for DC-DC converters, Expert Systems with Applications, Vol.36, No.2, 2009, pp.1540-1548.

[3] O. Cordn, A historical review of evolutionary learning methods for Mamdani-type fuzzy rule- 
Table 4. Results of GPFIS-Control: Cart-Centering Problem

\begin{tabular}{|c|c|c|c|c|}
\hline \multirow[b]{2}{*}{ Attribute } & \multicolumn{4}{|c|}{ Aggregation operator $=$ Max } \\
\hline & $\begin{array}{c}\text { Product+Root- } \\
\text { Sq }+ \\
\text { Height }\end{array}$ & $\begin{array}{c}\text { Product+Root-Sq+ } \\
\text { MaxHeight }\end{array}$ & $\begin{array}{c}\text { Product+Root-Sq+ } \\
\text { Neg+Height }\end{array}$ & $\begin{array}{c}\text { Product+Root-Sq+ } \\
\text { Neg+MaxHeight }\end{array}$ \\
\hline Average Steps $(0.02 s)$ & 215.9 & 243.6 & 224.6 & 203.5 \\
\hline Std. Dev. Steps $(0.02 s)$ & 25.73 & 94.09 & 37.89 & 60.78 \\
\hline Average Time (s) & $4.318 \mathrm{~s}$ & 4.872 & 4.492 & 4.07 \\
\hline Average Rules & 21 & 24 & 14 & 15 \\
\hline \multicolumn{5}{|c|}{ Aggregation operator $=$ Average } \\
\hline Attribute & $\begin{array}{c}\text { Product+Root- } \\
\text { Sq+ } \\
\text { Height }\end{array}$ & $\begin{array}{c}\text { Product+Root-Sq+ } \\
\text { MaxHeight }\end{array}$ & $\begin{array}{c}\text { Product+Root-Sq+ } \\
\text { Neg+Height }\end{array}$ & $\begin{array}{c}\text { Product+Root-Sq+ } \\
\text { Neg+MaxHeight }\end{array}$ \\
\hline Average Steps $(0.02 \mathrm{~s})$ & 160.2 & 135.8 & 205.5 & 144.9 \\
\hline Std. Dev. Steps $(0.02 s)$ & 18.92 & 18.94 & 38.99 & 11.43 \\
\hline Average Time (s) & 3.204 & 2.796 & 4.11 & 2.89 \\
\hline Average Rules & 27 & 28 & 26 & 24 \\
\hline
\end{tabular}

based systems. International Journal of Approximate Reasoning, Vol. 52, No. 6, 2011, pp.894-913.

[4] J. S. R. Jang, C. T. Sun, and E. Mizutani, Neuro-fuzzy and soft computing: a computational approach to learning and machine intelligence. Prentice-Hall, Englewood Cliffs, 1997.

[5] R.E. Precup, and H. Hellendoorn, A survey on industrial applications of fuzzy control, Computers in Industry, Vol. 62, No.3, 2011, pp.213-226.

[6] O. Cordon, F. Gomide, F. Herrera, F. Hoffmann, and L. Magdalena, Ten years of genetic fuzzy systems: current framework and new trends, Fuzzy Sets \& Systems, Vol.141, No.1, 2004, pp. 5-31.

[7] C. Karr, Genetic algorithms for fuzzy controllers, AI Expert, Vol.6, No. 2, 1991, pp.26-33.

[8] B. D. Liu, C. Y. Chen, and J. Y. Tsao, Design of adaptive fuzzy logic controller based on linguistic-hedge concepts and genetic algorithms, IEEE Transactions on Systems, Man and Cybernetics, Part B: Vol.31, No.1, 2001, pp.32-53.

[9] F. Herrera, M. Lozano, and J. L. Verdegay, A learning process for fuzzy control rules using genetic algorithms, Fuzzy Sets and Systems, Vol. 100, No. 1, 1998, pp.143-158.

[10] T. Pal, and N. R. Pal, SOGARG: A self-organized genetic algorithm-based rule generation scheme for fuzzy controllers. IEEE Transactions on Evolutionary Computation, Vol.7, No.4, 2003, pp.397415.

[11] E. Tunstel, and M. Jamshidi, On genetic programming of fuzzy rule-based systems for intelligent control, International Journal of Intelligent Automation and Soft Computing, Vol. 2, No. 3, 1996, pp.271-284.
[12] A. Tsakonas, Local and global optimization for Takagi-Sugeno fuzzy system by memetic genetic programming, Expert Systems with Applications, Vol.40, No.8, 2013, pp.3282-3298.

[13] N. Kasabov, and Q. Song, DENFIS: dynamic evolving neural-fuzzy inference system and its application for time-series prediction, IEEE Trans. Fuzzy Systems, Vol.10, No. 2, 2002, pp.144-154.

[14] R. J. Contreras, M.M.B.R. Vellasco, and R. Tanscheit, Hierarchical type-2 neuro-fuzzy BSP model, Information Sciences, Vol. 181, No. 15, 2011, pp. 3210-3224.

[15] M. P. Hinchliffe, M. J. Willis, H. Hiden, M.T. Tham, B. McKay, and G.W. Barton, Modeling chemical process systems using a multi-gene genetic programming algorithm, In: Proceedings of the First Annual Conference of Genetic Programming, J. R. Koza, MIT Press, Massachussets, 1996, pp. 56-65.

[16] D. P. Searson, M. J. Willis, and G.A. Montague, Co-evolution of non-linear PLS model components, Journal of Chemometrics, Vol. 2, 2007, pp. 592-603.

[17] F. Herrera, Genetic fuzzy systems: taxonomy, current research trends and prospects, Evolutionary Intelligence, Vol.1, No.1, 2008, pp.27-46.

[18] O. Castillo, and P. Melin, A review on the design and optimization of interval type-2 fuzzy controllers, Applied Soft Computing, Vol.12, No.4, 2012, pp.1267-1278.

[19] M. Fazzolari, R. Alcal, Y. Nojima, H. Ishibuchi, and F. Herrera, A Review of the Application of Multiobjective Evolutionary Fuzzy Systems: Current Status and Further Directions, IEEE Transactions on Fuzzy Sets, Vol.21, No.1, 2013, pp.45-65. 
[20] C. F. Juang, J. Y. Lin, and C. T. Lin, Genetic reinforcement learning through symbiotic evolution for fuzzy controller design, IEEE Transactions on Systems, Man, and Cybernetics, Part B, Vol.30, No.2, 2000, pp.290-302.

[21] E. De Santis, A. Rizzi, A. Sadeghiany, and F. M. F. Mascioli, Genetic optimization of a fuzzy control system for energy flow management in microgrids, In: Proceedings of IFSA World Congress and NAFIPS Annual Meeting, W. Pedrycz and M. Reformat, IEEE, New Jersey, 2013, pp. 418-423.

[22] L. H. Hassan, M. Moghavvemi, H. A. Almurib, O. Steinmayer, Application of genetic algorithm in optimization of unified power flow controller parameters and its location in the power system network, International Journal of Electrical Power \& Energy Systems, Vol.46, 2013, pp.89-97.

[23] R. P. Prado, S. Garca-Galn, J. Exposito, and A. J. Yuste, Knowledge acquisition in fuzzy-rule-based systems with particle-swarm optimization, IEEE Transactions on Fuzzy Systems, Vol.18, No.6, 2010, pp.1083-1097.

[24] O. Castillo, R. Martnez-Marroqun, P. Melin, F. Valdez, and J. Soria, Comparative study of bioinspired algorithms applied to the optimization of type-1 and type-2 fuzzy controllers for an autonomous mobile robot, Information Sciences, Vol.192, 2012, pp.19-38.

[25] E. Alba, C. Cotta, and J. M. Troya, Typeconstrained genetic programming for rule-base definition in fuzzy logic controllers, In: Proceedings of the First Annual Conference of Genetic Programming, J. R. Koza, MIT Press, Massachussets, 1996, pp. 255-260.

[26] E. Tunstel, and M. Jamshidi, On genetic programming of fuzzy rule-based systems for intelligent control, International Journal of Intelligent $\mathrm{Au}$ tomation and Soft Computing, Vol.2, No.3, 1996, pp.271-284.
[27] A. Homaifar, D. Battle, E. Tunstel, and G. Dozier, Genetic Programming Design of Fuzzy Logic Controllers for Mobile Robot Path Tracking, International Journal of Knowledge Based Intelligent Engineering Systems, Vol.4, No.1, 2000, pp.33-52.

[28] J. R. Koza, Genetic Programming: On the Programming of Computers by Means of Natural Selection, MIT Press, Massachusetts, 1992.

[29] W. B. Langdon, and R. Poli, Foundations of Genetic Programming, Springer-Verlag, Heidelberg, 2002.

[30] G. J. Klir, and B. Yuan, Fuzzy sets and fuzzy logic, Prentice-Hall, New Jersey, 1995.

[31] T. Calvo, A. Kolesrov, M. Komornkov, and R. Mesiar, Aggregation operators: properties, classes and construction methods, In: Aggregation Operators, T. Calvo et al., Physica-Verlag, Heidelberg, 2002, pp.3-104.

[32] R. R. Yager, J. Kacprzyk, and G. Beliakov, Recent developments in the ordered weighted averaging operators: theory and practice, Springer, Heidelberg, 2011.

[33] S. Luke and L. Panait, Lexicographic parsimony pressure, In: Proceedings of the Genetic and Evolutionary Computation Conference, W. B. Langdon et al., Morgan Kaufmann Publishers, New York, 2002, pp. 829-836.

[34] MATLAB 7.10.0 (R2010a), The MathWorks Inc, Massachusetts, 2010.

[35] P. R. Thrift, Fuzzy Logic Synthesis with Genetic Algorithms. In: Proceedings of the International Conference on Genetic Algorithms, R. K. Belew and L. B. Booker, Morgan Kauffman Publishers, California, pp. 509-513, July 1991. 